\title{
Connectivity reflects coding: a model of voltage-based STDP with homeostasis
}

\author{
Claudia Clopath $^{1}$, Lars Büsing ${ }^{1,2}$, Eleni Vasilaki ${ }^{1,2} \&$ Wulfram Gerstner ${ }^{1}$
}

\begin{abstract}
Electrophysiological connectivity patterns in cortex often have a few strong connections, which are sometimes bidirectional, among a lot of weak connections. To explain these connectivity patterns, we created a model of spike timing-dependent plasticity (STDP) in which synaptic changes depend on presynaptic spike arrival and the postsynaptic membrane potential, filtered with two different time constants. Our model describes several nonlinear effects that are observed in STDP experiments, as well as the voltage dependence of plasticity. We found that, in a simulated recurrent network of spiking neurons, our plasticity rule led not only to development of localized receptive fields but also to connectivity patterns that reflect the neural code. For temporal coding procedures with spatio-temporal input correlations, strong connections were predominantly unidirectional, whereas they were bidirectional under rate-coded input with spatial correlations only. Thus, variable connectivity patterns in the brain could reflect different coding principles across brain areas; moreover, our simulations suggested that plasticity is fast.
\end{abstract}

Experience-dependent changes in receptive fields ${ }^{1,2}$ or in learned behavior relate to changes in synaptic strength. Electrophysiological measurements of functional connectivity patterns in slices of neural tissue ${ }^{3,4}$ or anatomical connectivity measures can only present a snapshot of the momentary connectivity, which may change over time ${ }^{5}$. The question then arises of whether the connectivity patterns and their changes can be connected to basic forms of synaptic plasticity ${ }^{6}$ such as long-term potentiation (LTP) and depression (LTD $)^{7}$. LTP and LTD depend on the exact timing of pre- and postsynaptic action potentials ${ }^{2-8}$ but also on postsynaptic voltage $\mathrm{e}^{9,10}$ and presynaptic stimulation frequency ${ }^{11}$. STDP has attracted particular interest in recent years, as temporal coding schemes in which information is contained in the exact timing of spikes rather than mean frequency can be learned by a neural system using STDP ${ }^{12,13}$ (review in ref. 14). However, the question of whether STDP is more fundamental than frequency-dependent plasticity or voltage-dependent plasticity rules has not been resolved despite an intense debate ${ }^{15}$. Moreover, it is unclear how the interplay of coding and plasticity yields the functional connectivity patterns that are seen in experiments. In particular, the presence or absence of bidirectional connectivity between cortical pyramidal neurons seems to be contradictory across experimental preparations in visual ${ }^{3}$ or somatosensory cortex ${ }^{4}$. Recent experiments have shown that STDP is strongly influenced by postsynaptic voltage before action potential firing ${ }^{16}$ but were unable to answer the question of whether spiketiming dependence is a direct consequence of voltage dependence or the manifestation of an independent process. In addition, STDP depends on stimulation frequency ${ }^{16}$, suggesting an interaction between timing- and frequency-dependent processes $^{16}$.

We found that a simple Hebbian plasticity rule that pairs presynaptic spike arrival with the postsynaptic membrane potential was sufficient to explain STDP and the dependence of plasticity on presynaptic stimulation frequency. Moreover, the intricate interplay of voltage and spike timing as well as the frequency dependence of STDP can be explained in our model from one single principle. In contrast with earlier attempts towards a unified description of synaptic plasticity ${ }^{17,18}$, our model is a phenomenological one. It does not give an explicit interpretation in terms of biophysical quantities such a calcium concentration ${ }^{17}, \mathrm{CaMKII}^{18}$, glutamate binding, NMDA receptors, etc. Instead, it aims at a minimal description of the major phenomena observed in electrophysiology experiments. The advantage of such a minimal model is that it allows us to discuss functional consequences in small ${ }^{19-21}$, and possibly even large ${ }^{22,23}$, networks. We found that the learning rule led to input specificity in small networks of up to ten neurons, which is necessary for receptive field development, similar to earlier models of STDP ${ }^{12,19}$ or ratebased plasticity rules ${ }^{24,25}$. We explicitly addressed the question of whether functional connectivity patterns of cortical pyramidal neurons measured in recent electrophysiological studies ${ }^{3,4}$ could be the result of plasticity during continued stimulation of neuronal model networks, particularly bidirectional connections ${ }^{3}$ that are incompatible with standard STDP models ${ }^{12,19}$. The mathematical simplicity of our model enabled us to identify conditions under which it becomes equivalent to the well-known Bienenstock-Cooper-Munro (BCM) model ${ }^{24}$ used in classical rate-based descriptions of developmental learning and, similar to some earlier models of STDP ${ }^{26,27}$, and why our model is fundamentally different from classical STDP models ${ }^{12,14,19}$, is widely used for temporal coding.

\section{RESULTS}

To study the means by which connectivity patterns in cortex can emerge from plasticity, we needed a plasticity rule that was consistent

\footnotetext{
${ }^{1}$ Laboratory of Computational Neuroscience, Brain-Mind Institute and School of Computer and Communication Sciences, Ecole Polytechnique Fédérale de Lausanne, Lausanne, Switzerland. 2Present address: Institut für Grundlagen der Informationsverarbeitung, Graz University of Technology, Austria (L.B.), and Department of Computer Science, University of Sheffield, Sheffield, UK (E.V.). Correspondence should be addressed to C.C. (claudia.clopath@epfl.ch).
} 
Figure 1 Illustration of the model. Synaptic weights react to presynaptic events (top) and postsynaptic membrane potential (bottom). (a) The synaptic weight was decreased if a presynaptic spike $x$ (green) arrived when the low-pass-filtered value $\bar{u}_{-}$(magenta) of the membrane potential was above $\theta_{-}$(dashed horizontal line). (b) The synaptic weight was increased if the membrane potential $u$ (black) was above a threshold $\theta_{+}$and the low-pass-filtered value of the membrane potential $\bar{u}_{+}$(blue) was higher than a threshold $\theta_{-}$and the presynaptic low-pass filter $\bar{x}$ (orange) was nonzero. (c) Step current injection made the postsynaptic neuron fire at $50 \mathrm{~Hz}$ in the absence of presynaptic stimulation (membrane potential $u$ in black). No weight change was observed. Note the depolarizing spike afterpotential, consistent with experimental data. (d) Reproduced from ref. 16. (e-h) Voltage-clamp experiment. A neuron received weak presynaptic stimulation of $2 \mathrm{~Hz}$ during $50 \mathrm{~s}$ while the postsynaptic voltage was clamped to values between $-60 \mathrm{mV}$ and $0 \mathrm{mV}$. (e-g) Schematic drawing of the trace $\bar{x}$ (orange) of the presynaptic spike train (green) as well as the voltage (black) and the synaptic weight (blue) for hyperpolarization (e), slight depolarization (f) and large depolarization (g). (h) The weight change as a function of clamped voltage using the standard set of parameters for visual cortex data (dashed blue line, voltage paired with 25 spikes at the synapse). With a different set of parameters, the model fit the experimental data (red circles) in hippocampal slices ${ }^{10}$ (see Online Methods for details). a
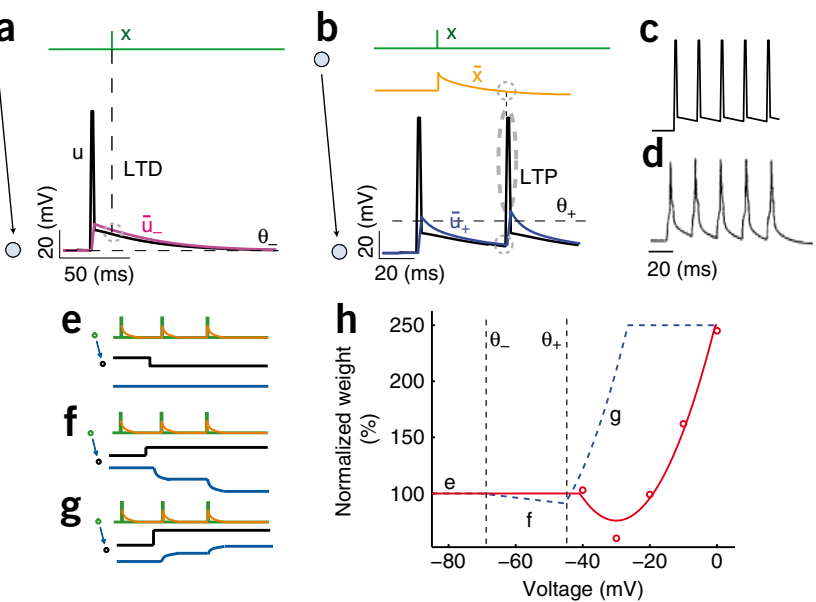

with a large body of experimental data. Because synaptic depression and potentiation occur via different pathways ${ }^{28}$, our model used separate additive contributions to the plasticity rule, one for LTD and another one for LTP (see Fig. 1 and Online Methods).

\section{Fitting the plasticity model to experimental data}

Consistent with voltage-clamp ${ }^{10}$ and stationary-depolarization experiments ${ }^{9}$, LTD is triggered in our model if presynaptic spike arrival occurs while the membrane potential of the postsynaptic neuron is slightly depolarized (above a threshold $\theta_{-}$that is usually set to resting potential), whereas LTP occurs if depolarization is big (above a second threshold $\theta_{+} ;$Fig. 1). The mathematical formulation of the plasticity rule makes a distinction between the momentary voltage $u$ and the low-pass-filtered voltage variables $\bar{u}_{-}$or $\bar{u}_{+}$, which denote temporal averages of the voltage over the recent past $\left(\bar{u}_{-}\right.$and $\bar{u}_{+}$indicate filtering of $u$ with two different time constants). Similarly, the event $x$ of presynaptic spike arrival needs to be distinguished from the trace $\bar{x}$ that is left at the synapse after stimulation by neurotransmitter. Potentiation occurs only if the momentary voltage is above $\theta_{+}$(this condition is fulfilled during action potential firing) and the average voltage $\bar{u}_{+}$is above $\theta_{-}$(this is fulfilled if there was a depolarization in the recent past) and the trace $\bar{x}$ left by a previous presynaptic spike event is nonzero (this condition holds if a presynaptic spike arrived a few milliseconds earlier at the synapse; Fig. 1b). LTD occurs if the average voltage $\bar{u}_{-}$is above $\theta_{-}$at the moment of a presynaptic spike arrival (Fig. 1a). The amount of LTD in our model depended on a homeostatic process on a slower time scale ${ }^{29}$. Low-pass filtering of the voltage by the variable $\left(\bar{u}_{-}\right.$or $\left.\bar{u}_{+}\right)$refers to some unidentified intracellular processes triggered by depolarization, for example, increase in calcium concentration or second messenger chains. Similarly, the biophysical nature of the trace $\bar{x}$ is irrelevant for the functionality of the model, but a good candidate process is the fraction of glutamate bound to postsynaptic receptors.

We used a STDP protocol in which presynaptic spikes arrive a few milliseconds before or after a postsynaptic spike (Fig. 2 and Supplementary Methods). If a post-pre pairing with a timing difference of $10 \mathrm{~ms}$ was repeated at frequencies below $35 \mathrm{~Hz}$, LTD occurred
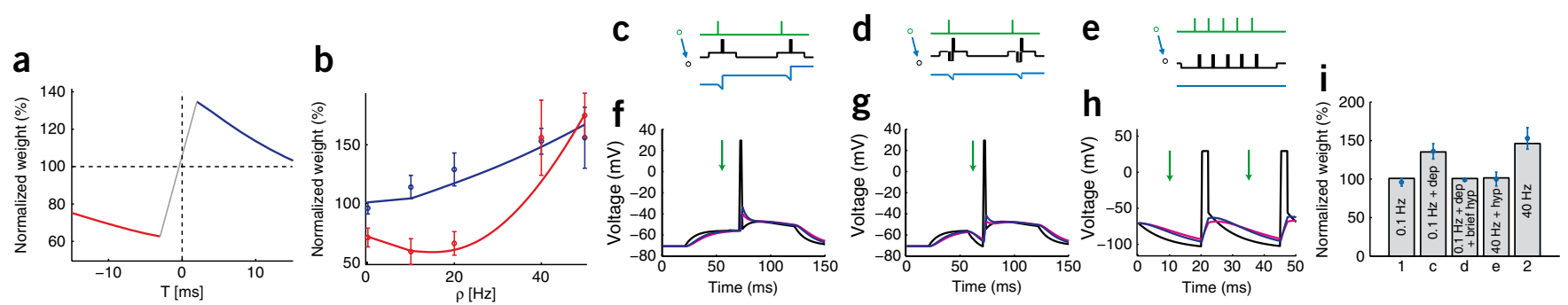

Figure 2 Fitting the model to experimental data. (a,b) Simulated STDP experiments. (a) Spike timing-dependent learning window: synaptic weight change for different time intervals $T$ between pre- and postsynaptic firing using 60 pre-post-pairs at $20 \mathrm{~Hz}$. (b) Weight change as a function of pairing repetition frequency $\rho$ using pairings with a time delay of $+10 \mathrm{~ms}$ (pre-post, blue) and $-10 \mathrm{~ms}$ (post-pre, red). Dots represent data from ref. 16 and lines represent our plasticity model. (c-i) Interaction of voltage and STDP. (c-e) Schematic induction protocols (green, presynaptic input; black, postsynaptic current; blue, evolution of synaptic weight). (c) Low-frequency potentiation is rescued by depolarization ${ }^{16}$. Low-frequency $(0.1 \mathrm{~Hz})$ pre-post spike pairs yielded LTP if a 100-ms-long depolarized current was injected around the pairing. (d) LTP failed if an additional brief hyperpolarized pulse was applied $14 \mathrm{~ms}$ before postsynaptic firing so that voltage is brought to rest. (e) Hyperpolarization preceding action potential prevents potentiation that normally occurred at $40 \mathrm{~Hz}^{16}$. (f) The simulated postsynaptic voltage $u$ (black) is shown after using the protocol described in c, together with temporal averages $\bar{u}_{-}$(magenta) and $\bar{u}_{+}$(blue). The presynaptic spike time is indicated by the green arrow. Using the model (equation (3)) with this setting yielded potentiation. (g) Data are presented as in $\mathbf{f}$ but using the protocol described in $\mathbf{d}$. No weight change was measured. (h) Data are presented as in $\mathbf{f}$ but using the protocol described in e. No weight change was measured. (i) Histogram summarizing the normalized synaptic weight of the simulation (bar) and the experimental data ${ }^{16}$ (dot, blue bar indicates variance) for $0.1-\mathrm{Hz}$ pairing (control 1), $0.1-\mathrm{Hz}$ pairing with the depolarization (protocol c), $0.1-\mathrm{Hz}$ pairing with the depolarization and brief hyperpolarization (protocol d), 40-Hz pairing (control 2), and 40- $\mathrm{Hz}$ pairing with the constant hyperpolarization (protocol e); parameters are described in Table 1. 

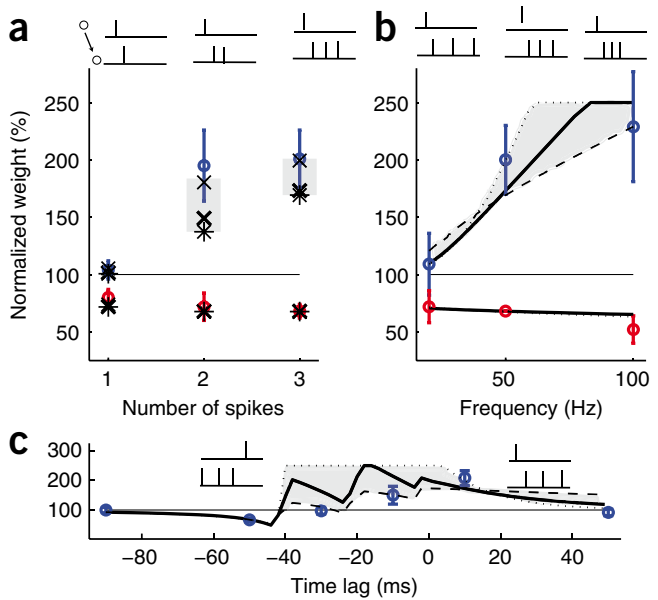

in our model (Fig. 2a,b), consistent with experimental data ${ }^{16}$. Repeated pre-post pairings (with 10 -ms timing difference) at frequencies above $10 \mathrm{~Hz}$ yielded LTP, but pairings at $0.1 \mathrm{~Hz}$ did not show any change in the model or in experiments ${ }^{16}$. In the model, these results can be explained by the fact that, at a $0.1-\mathrm{Hz}$ repetition frequency, the lowpass-filtered voltage $\bar{u}_{+}$, which increases abruptly during postsynaptic spiking, decays back to zero before the next impulse arrives; thus, LTP cannot be triggered. However, as LTD in the model requires only a weak depolarization of $\bar{u}_{-}$at the moment of presynaptic spike arrival, post-pre pairings give rise to depression, even at a very low frequency. At repetition frequencies of $50 \mathrm{~Hz}$, the post-pre procedure is nearly indistinguishable from a pre-post timing and LTP dominates.

If a pre-post protocol at $0.1 \mathrm{~Hz}$ that normally does not induce LTP was combined with a depolarizing current pulse, then potentiation was observed in experiments ${ }^{16}$ and in our model (Fig. 2c,f,i). As a result of the injected current, the low-pass-filtered voltage variable $\bar{u}_{+}$is depolarized before the pairing. Thus, at the moment of the postsynaptic spike, the average voltage $\bar{u}_{+}$is above the threshold $\theta_{-}$, leading to potentiation. Similarly, a pre-post protocol that normally leads to LTP can be blocked if the postsynaptic spikes are triggered on the background of a hyperpolarizing current (Fig. 2e,h,i).

To study nonlinear aspects of STDP, we simulated a protocol of burst timing-dependent plasticity in which presynaptic spikes are paired with 1-3 postsynaptic spikes ${ }^{30}$ (see Online Methods). Although pairings at $0.1 \mathrm{~Hz}$ did not change the synaptic weight, repeated triplets pre-post-post generated potentiation in our model, as the first postsynaptic spike induced a depolarizing spike after potential so that $\bar{u}_{+}$was depolarized. Adding a third postsynaptic

Figure 4 Weight evolution in an all-to-all connected network of ten neurons. (a) Rate code. Neurons fired at different frequencies, neuron 1 at $2 \mathrm{~Hz}$, neuron 2 at $4 \mathrm{~Hz}$, neuron 10 at $20 \mathrm{~Hz}$. The weights (bottom) averaged over $100 \mathrm{~s}$ indicate that neurons with high firing rates developed strong bidirectional connections (light blue, weak connections (under $2 / 3$ of the maximal value); yellow, strong unidirectional connections (above $2 / 3$ of the maximal value); brown, strong bidirectional connections). The cluster is schematically represented (after). (b) Temporal code. Neurons fired successively every 20 ms (neuron 1, followed by neuron $220 \mathrm{~ms}$ later, followed by neuron $320 \mathrm{~ms}$ later, etc). Connections (bottom) were unidirectional with strong connections from presynaptic neuron with index $n$ (vertical axis) to postsynaptic neuron with index $n+1, n+2$ and $n+3$, leading to a ring-like topology. (c,d) Data are presented as in $\mathbf{a}$ and $\mathbf{b}$, but we used a standard STDP rule $\mathrm{e}^{12,14,19}$. Bidirectional connections are impossible.
Figure 3 Burst timing-dependent plasticity. One presynaptic spike was paired with a burst of postsynaptic spikes. This pairing was repeated 60 times at $0.1 \mathrm{~Hz}$. (a) Normalized weight as a function of the number of postsynaptic spikes $(1,2,3)$ at $50 \mathrm{~Hz}$ (dots represent data from ref. 30 , crosses represent simulation). The presynaptic spike was paired +10 ms before the first postsynaptic spike (blue) or $-10 \mathrm{~ms}$ after (red). (b) Normalized weight as a function of the frequency between the three postsynaptic action potentials (dots indicate data, lines indicate simulation, blue indicates pre-post, red indicates post-pre). (c) Normalized weight as a function of the timing between the presynaptic spike and the first postsynaptic spike of a three-spike burst at $50 \mathrm{~Hz}$ (dot indicates data, black lines indicate simulation). A hard upper bound was set to $250 \%$ normalized weight. The dashed line and crosses and the dotted line and stars represent simulations with alternative sets of parameters, $A_{\mathrm{LTD}}=21 \times 10^{-5} \mathrm{mV}^{-1}, A_{\mathrm{LTP}}=50 \times 10^{-4} \mathrm{mV}^{-2}, \tau_{x}=143 \mathrm{~ms}$, $\tau_{-}=6 \mathrm{~ms}, \tau_{+}=5 \mathrm{~ms}$ and $A_{\mathrm{LTD}}=21 \times 10^{-5} \mathrm{mV}^{-1}, A_{\mathrm{LTP}}=67 \times 10^{-4} \mathrm{mV}^{-2}$, $\tau_{\mathrm{x}}=5 \mathrm{~ms}, \tau_{-}=8 \mathrm{~ms}, \tau_{+}=5 \mathrm{~ms}$, respectively. Shading indicates reachable data points generated by the model with different parameters.

spike to the protocol (that is, quadruplets pre-post-post-post) did not lead to stronger LTP (Fig. 3a). Our model also describes the dependence of LTP on the intra-burst frequency (Fig. 3b). At an intra-burst frequency of $20 \mathrm{~Hz}$, no LTP occurred because the second spike in the burst came so late that the presynaptic trace $\bar{x}$ had decayed back to zero. At higher intra-burst frequencies, the three conditions for LTP $\left(u(t)>\theta_{+}\right.$and $\bar{u}_{+}>\theta_{-}$and $\left.\bar{x}>0\right)$ are fulfilled. The burst-timing dependence (Fig. 3c) that occurs when the timing of one presynaptic spike is changed with respect to a burst of three postsynaptic spikes is qualitatively similar to that found experimentally 30,31 , but only four of the six experimental data points are quantitatively reproduced by the model with a given set of parameters. Notably, our model predicted that the curve of burst timing-dependent plasticity should show a change in the amount of potentiation whenever the presynaptic spike is shifted across one of the three postsynaptic spikes (Fig. 3c). Because dendritic spikes, which are relevant for burst timing-dependent $\mathrm{STDP}^{31}$, are broader than somatic action potentials, the 'jumps' in the burst-STDP curves would be blurred. a Rate code
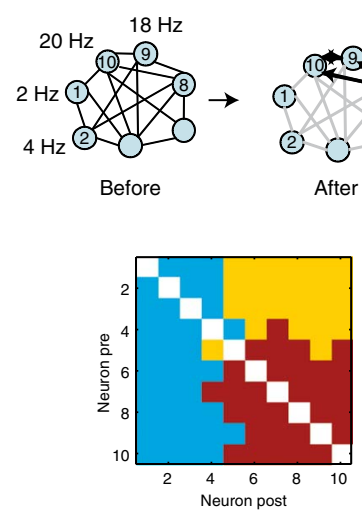

C

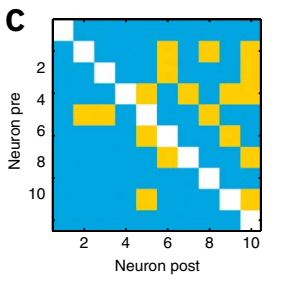

b

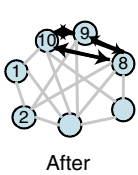

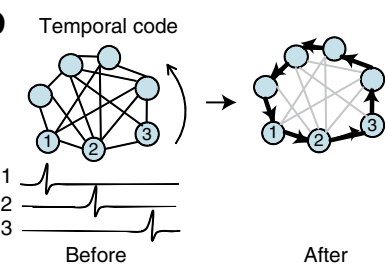

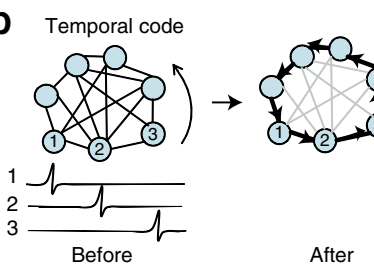

After
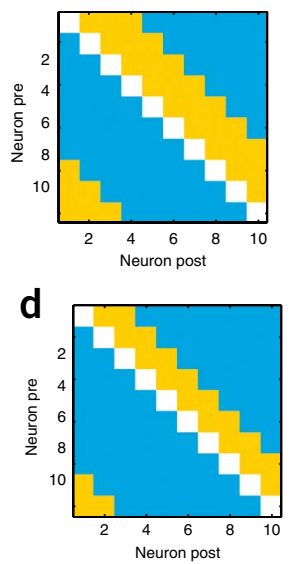
a

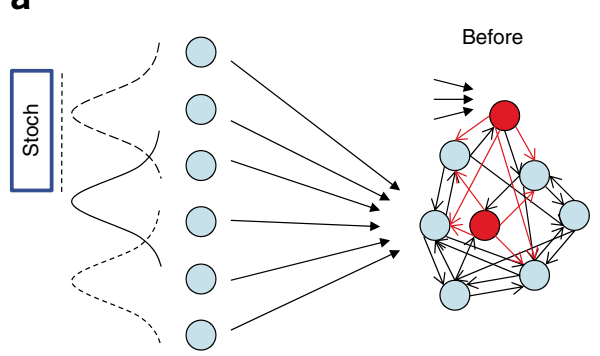

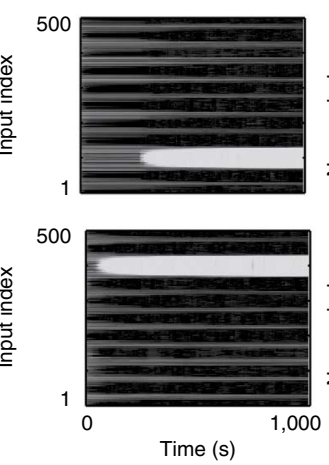
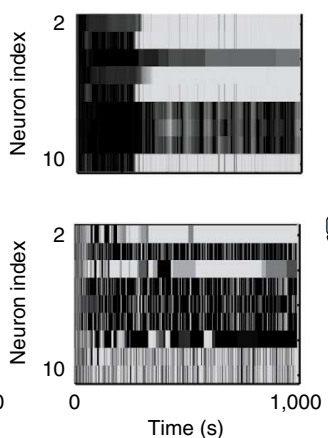
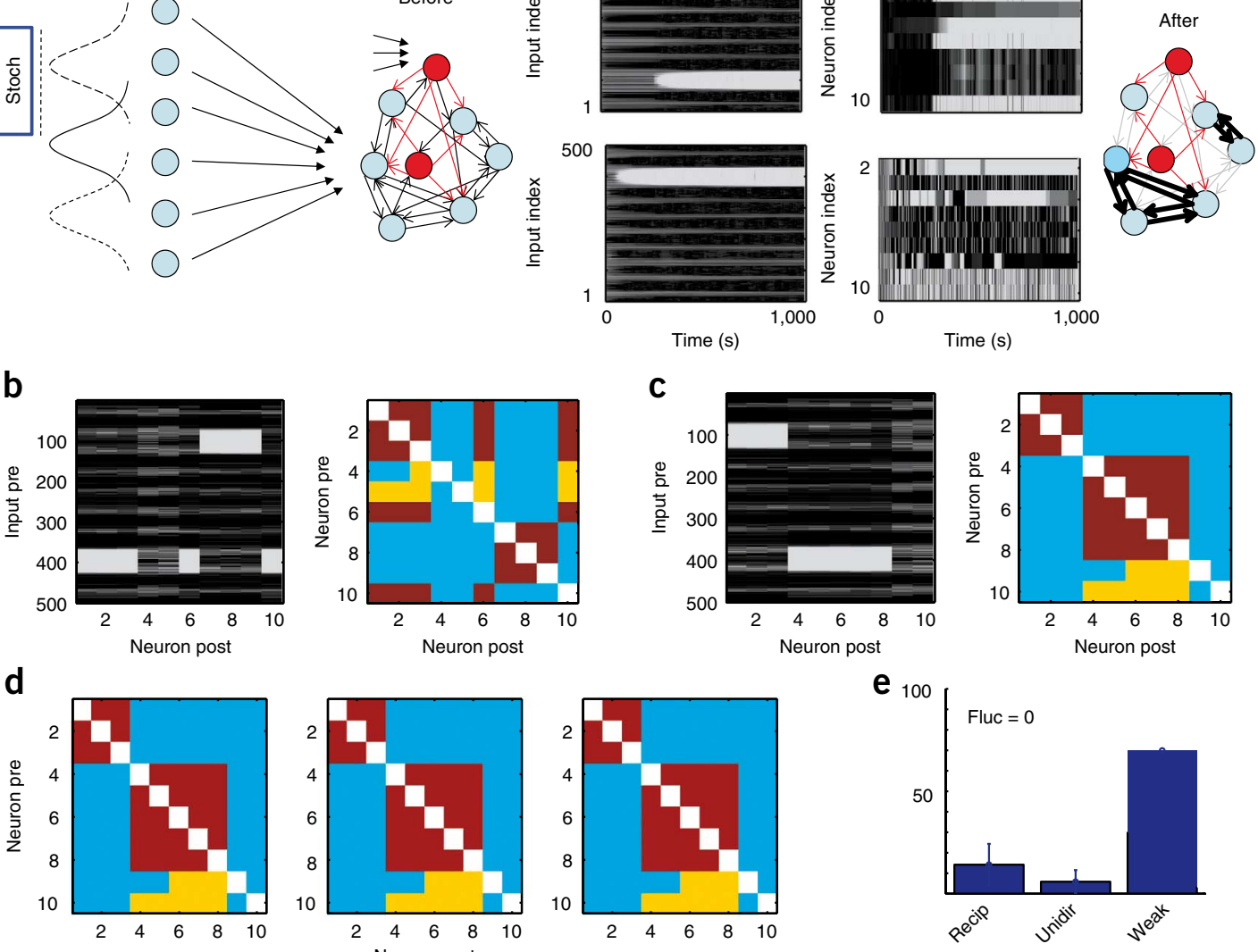

e 100
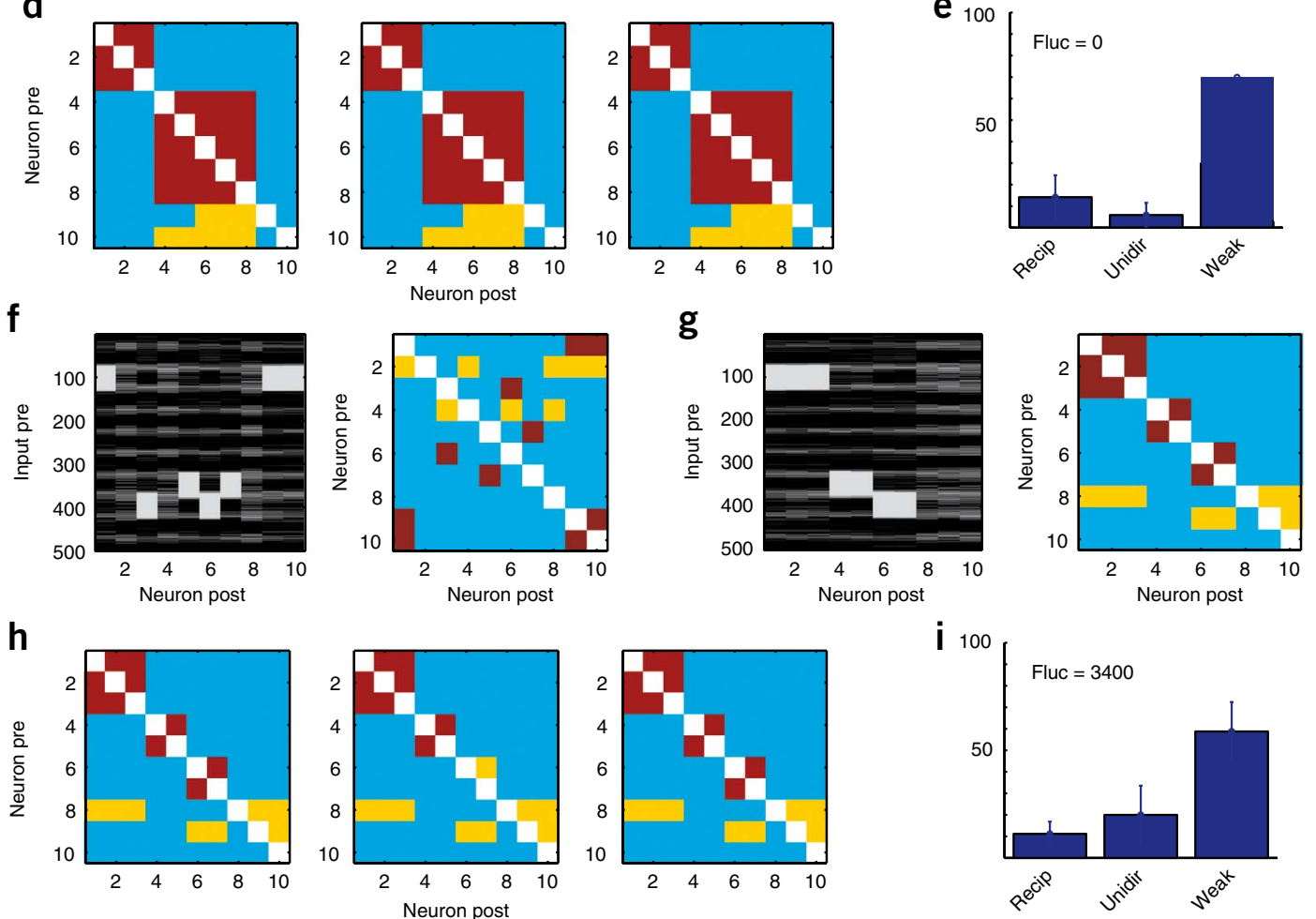

Figure 5 Plasticity during rate coding. (a) A network of ten excitatory (light blue) and three inhibitory neurons (red) received feedforward inputs from 500 Poisson spike trains with a Gaussian profile of firing rates. The center of the Gaussian was shifted randomly every 100 ms (schematic network before (left) and after the plasticity experiment (right)). The temporal evolution of the weights are shown (top, small amplitudes of plasticity; bottom, normal amplitudes of plasticity; left, feedforward connections onto neuron 1; right, recurrent connections onto neuron 1). (b-e) Learning with small amplitudes. We used the parameters detailed in Table $1 \mathbf{b}$ (visual cortex) except for the amplitudes $A_{\mathrm{LTP}}$ and $A_{\mathrm{LTD}}$, which were reduced by a factor 100 . (b) Mean feedforward weights (left) and recurrent excitatory weights (right) averaged over $100 \mathrm{~s}$. The feedforward weights (left) indicate that the neurons developed localized receptive fields (light gray). The recurrent weights (right) were classified as weak (less than $2 / 3$ of the maximal weight, light blue), strong unidirectional (more than $2 / 3$ of the maximal weight, yellow) or strong reciprocal (brown) connections. The diagonal is white, as self-connections do not exist in the model. (c) Data are presented as in $\mathbf{b}$, but the neuron index was reordered. (d) Three snapshots of the recurrent connections taken $5 \mathrm{~s}$ apart indicate that recurrent connections were stable. (e) Histogram of reciprocal, unidirectional and weak connections in the recurrent network averaged over $100 \mathrm{~s}$, as shown in $\mathbf{b}$ (fluc, fluctuations). The total number of weight fluctuations during $100 \mathrm{~s}$ was zero. The histogram shows an average of ten repetitions (error bars represent s.d.). ( $\mathbf{f}-\mathbf{i})$ Rate code during learning with normal amplitudes. We used the network described above but with a standard set of parameters (Table $1 \mathbf{b}$, visual cortex). (f) Receptive fields were localized. (g) Reordering showed clusters of neurons with bidirectional coupling. These clusters were stable when averaged over $100 \mathrm{~s}$. (h) Connections were able to change from one time step to the next. (i) The percentage of reciprocal connections was high, but because of fluctuations, more than 1,000 transitions between strong unidirectional to strong bidirectional or back occurred during $100 \mathrm{~s}$. 
a
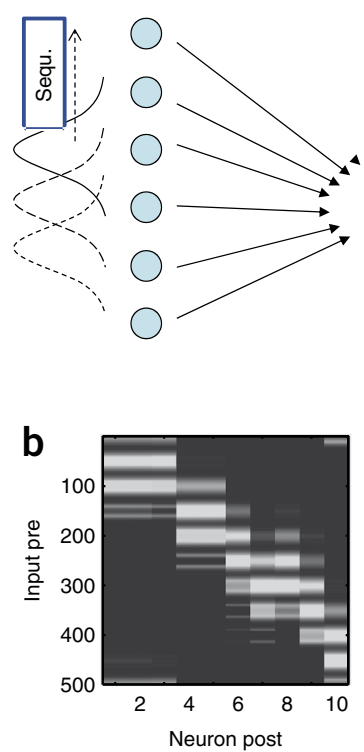

d

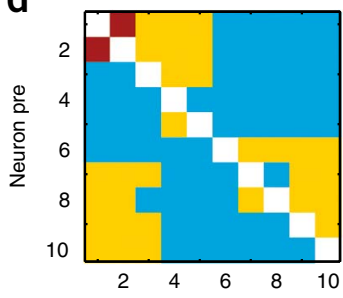

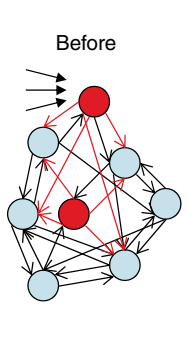

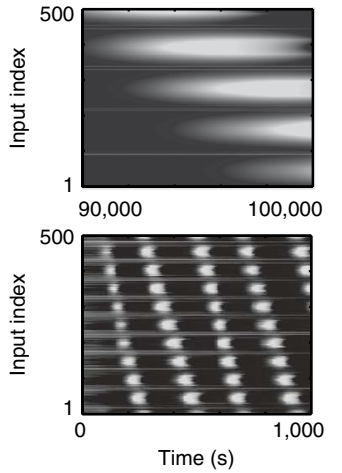

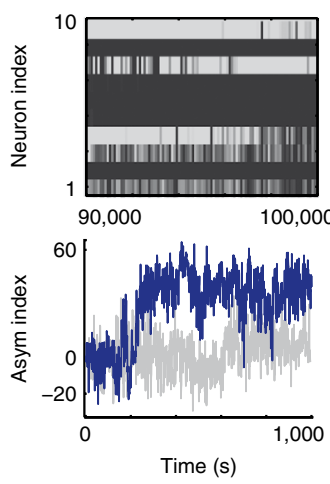
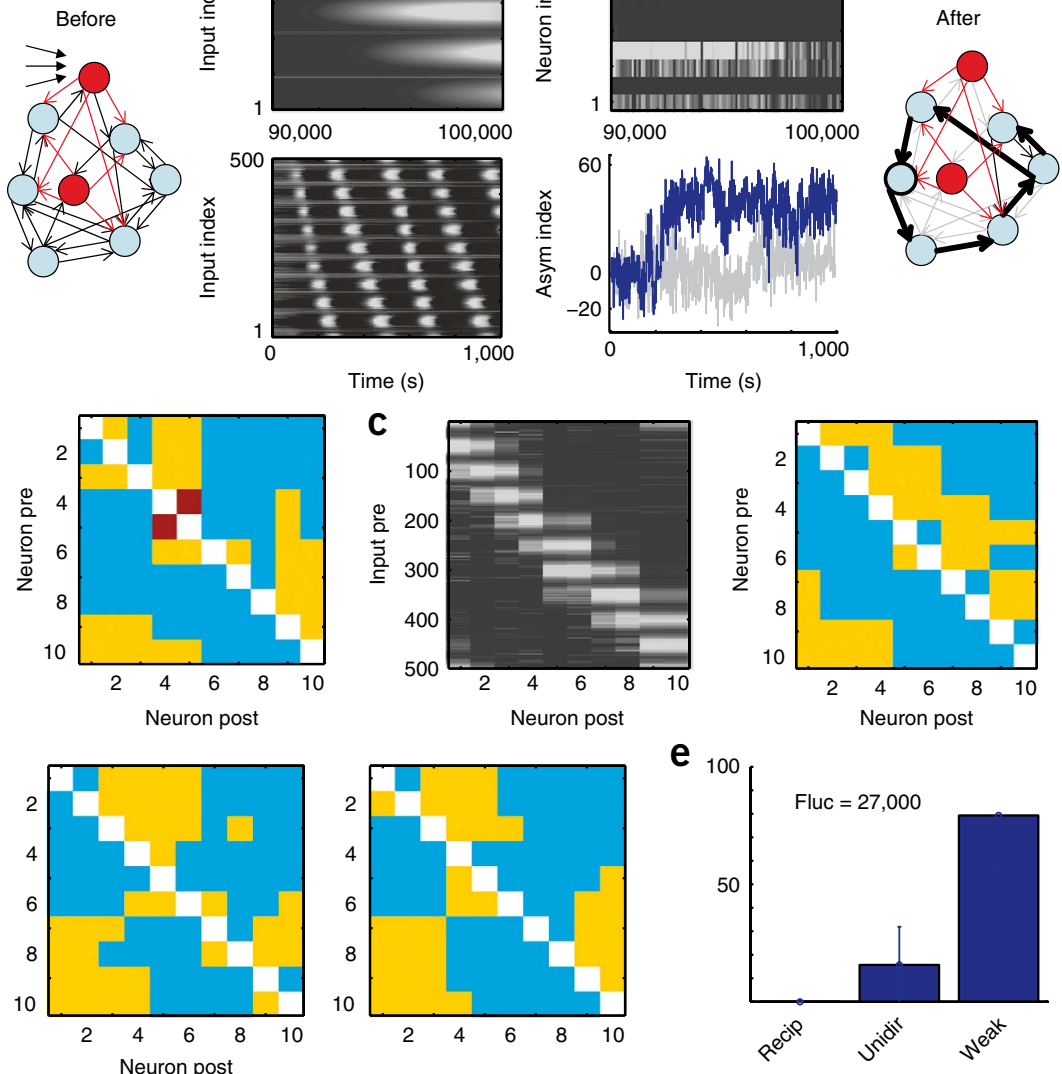

C
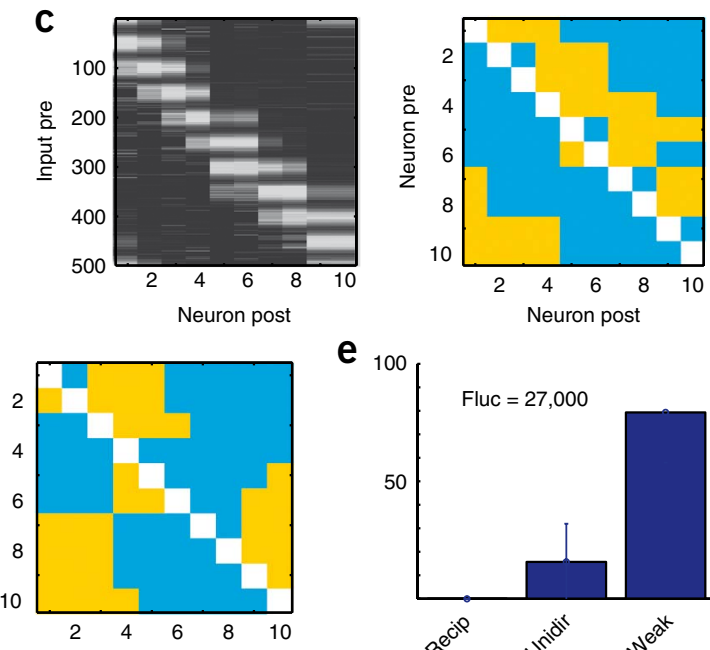

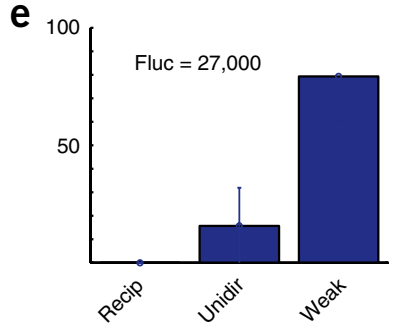

Figure 6 Temporal-coding procedure. The same parameters were used as in Figure $\mathbf{5}$ (Table $\mathbf{1 b}$, visual cortex), but input patterns were moved successively every $20 \mathrm{~ms}$, corresponding to a step-wise motion of the Gaussian stimulus profile across the input neurons. (a) The schematic figure shows the network before and after the plasticity experiment. Shown are the temporal evolution of the weights (top panels: amplitude of synaptic plasticity for feedforward connections reduced by a factor of 100; left, feedforward weights onto neuron 6; right, lateral connections onto neuron 6 ; bottom panels: normal amplitude of plasticity; left, feedforward connections onto neuron 1 ; right, temporal evolution of asymmetry index of connection pattern (gray line indicates asymmetrical index for simulation; Fig. 5). Positive values indicate the weights from neurons $n$ to $n+k$ are stronger than those from $n$ to $n-k$ for $1 \leq k \leq 3$ ). (b) Receptive fields are localized (left). The recurrent network developed a ring-like structure with strong unidirectional connections from neuron 8 (vertical axis) to neurons 9 and 10 (horizontal axis), etc. (small amplitudes of plasticity). (c) Data are presented as in b, but normal plasticity values were used. (d) Some of the strong unilateral connections appeared or disappeared from one time step to the next, but the ring-like network structure persisted, as the lines just above the diagonal are much more populated than the line below the diagonal. (e) Reciprocal connections are absent, but unidirectional connections fluctuated several times between weak and strong during $100 \mathrm{~s}$.

\section{Functional implications}

Connectivity patterns in a local cortical circuit have been shown to be nonrandom; that is, the majority of connections are weak and the rare strong ones have a high probability of being bidirectional ${ }^{3}$. However, standard models of STDP ${ }^{14}$ do not exhibit stable bidirectional connections ${ }^{19,32}$. Intuitively, if cell A fires before cell B, a pre-post pairing for the $\mathrm{AB}$ connection is formed so that the connection is strengthened. The post-pre pairing occurring at the same time in the BA connection leads to depression. It is therefore impossible to strengthen both connections at the same time. Moreover, to assure long-term stability of firing rates, parameters in standard STDP rules are typically chosen such that inhibition slightly dominates excitation $^{14}$, which implies that random spike firing decreases connections. However, the nonlinear aspects of plasticity in our model changed such a simple picture. From the results (Figs. $2 \mathbf{b}$ and $\mathbf{3 b}$ ), we expect that our model could develop stable bidirectional connections at higher neuronal firing rates, in contrast with standard STDP rules.

We first simulated a small network of ten all-to-all connected neurons in which each neuron fired at a fixed frequency, but the frequency varied across neurons. We found that bidirectional connections were formed only between those neurons that both fired at a high rate (Fig. 4a). In a second simulation, the neurons in the same network were stimulated cyclically such that they fired in a distinct temporal order $(1,2,3, \ldots)$. In this case, the weights form, after a period of synaptic plasticity, a loop in which strong connections from 1 to 2, 2 to $3 \ldots$ develop but bidirectional connections do not (Fig. 4b). These results contrast with those of simulation experiments using a standard STDP rule, where connections are always unidirectional, independently of the stimulation procedure (Fig. 4c,d). Theoretical arguments (Supplementary Methods) indicate that bidirectional connections cannot exist under the cyclic temporal stimulation procedure (for standard STDP or for our plasticity model). Bidirectional connections did develop in our nonlinear voltage-dependent plasticity model under the assumption of slowly varying rates, in contrast with standard STDP (Fig. $\mathbf{4 c}, \mathbf{d})$.

To move to a more realistic scenario, we simulated a network of ten excitatory neurons (with all-to-all connectivity) and three inhibitory neurons. Each inhibitory neuron received input from eight randomly selected excitatory neurons and randomly projected 
Figure 7 Receptive fields development. (a) A small patch of $16 \times 16$ pixels was chosen from the whitened natural images benchmark ${ }^{35}$. The patch was selected randomly and was presented as input to 512 neurons for $200 \mathrm{~ms}$. The positive part of the image was used as the firing rate to generate Poisson spike trains of the 256 ON inputs and the negative one for the 256 OFF inputs. (b) The weights after convergence are shown for the ON inputs and the OFF inputs rearranged on a $16 \times 16$ image. The filter was calculated by subtracting the OFF weights from the $\mathrm{ON}$ weights. The filter was localized and bimodal, corresponding to an oriented receptive field. (c) Temporal evolution of the weights shown in the red dashed box in b. (d) Nine different neurons. (e) Two different neurons receiving presynaptic input with varying firing rates from $0-25 \mathrm{~Hz}$ (top), 0-37.5 Hz (middle) and $0-75 \mathrm{~Hz}$ (bottom).

back to six excitatory neurons. In addition to the recurrent input, each excitatory and inhibitory neuron received feedforward spike input from a

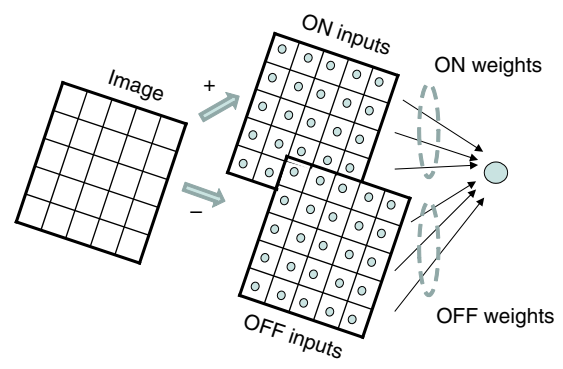

C

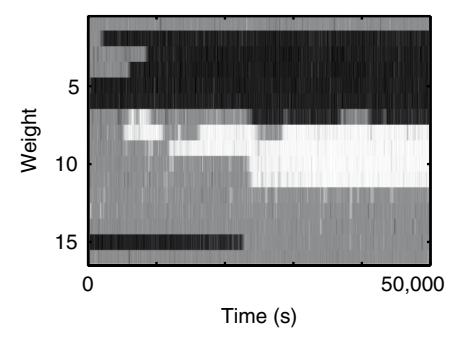

b

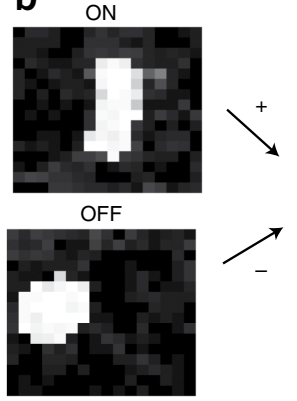

d

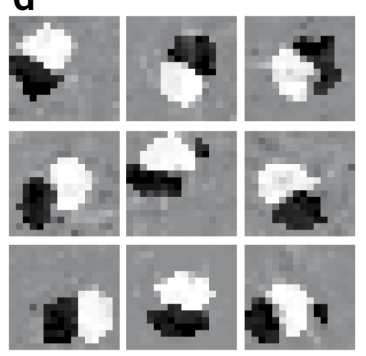

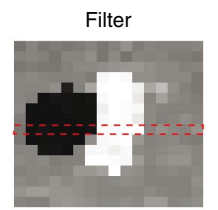

e

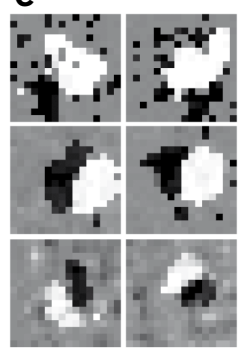
500 presynaptic neurons $j$ that generated stochastic Poisson input at a rate $v_{j}$. The rates of neighboring input neurons were correlated, mimicking the presence or absence of spatially extended objects. The location of the stimulus was switched every $100 \mathrm{~ms}$ to a new random position. In case of retinal input, this would correspond to a situation where the subject fixates every $100 \mathrm{~ms}$ on a new stationary stimulus. Depending on the retinal position of stimulus, a given postsynaptic neuron responds with a low, medium or high firing rate, which is stationary during the 100 -ms stimulation period; the firing rates of the ten neurons in the network encode the current position of the stimulus (rate-coding procedure). In a temporal-coding procedure, the model input is shifted every $20 \mathrm{~ms}$ to a neighboring location, mimicking rapid movement of an object across an array of sensory receptors (for example, during whisking behavior) ${ }^{33}$. In this scenario, a given model neuron exhibits only short, transient

bursts of a few spikes; thus, it is the temporal structure of the activity (as opposed to stationary firing rates) that encodes the position and movement of the stimulus. For both scenarios, the network is identical. Feedforward connections and lateral connections between model pyramidal neurons are plastic, whereas connections to and from inhibitory neurons are fixed.

During the first 100-400 s of stimulation in the rate-coding procedure, the excitatory neurons developed localized receptive fields; that is, weights from neighboring inputs to the same postsynaptic neuron became either strong or weak together and stayed stable thereafter (Fig. 5a). Similarly, lateral connections onto the same postsynaptic neuron developed to strong or weak synapses, which remained, apart from fluctuations, stable thereafter (Fig. 5a), leading to a structured pattern of synaptic connections (Fig. 5b). After reordering from the neurons according to similarity of receptive fields, we found that three

Table 1 Parameters

a

Parameters

Value

$C$, membrane capacitance

$g_{L}$, leak conductance

$E_{L}$, resting potential

$\Delta_{T}$, slope factor

$V_{T_{\text {rest }} \text {, threshold potential at rest }}$

$\tau_{w_{a d} \text {, adaptation time constant }}$

a, subthreshold adaptation

$b$, spike triggered adaptation

Isp, spike current after a spike

$\tau_{z}$, spike current time constant

$\tau_{V_{T}}$, threshold potential time constant

VTmax, threshold potential after a spike

$281 \mathrm{pF}$
$30 \mathrm{nS}$
$-70.6 \mathrm{mV}$
$2 \mathrm{mV}$
$-50.4 \mathrm{mV}$
$144 \mathrm{~ms}$
$4 \mathrm{nS}$
$0.805 \mathrm{pA}$
$400 \mathrm{pA}$
$40 \mathrm{~ms}$
$50 \mathrm{~ms}$
$30.4 \mathrm{mV}$

b

Experiments

Visual cortex*,16

Somatosensory cortex 30

Hippocampal 10

$\begin{array}{ll}\theta_{-}(\mathrm{mV}) & \theta_{+}(\mathrm{mV} \\ -70.6 & -45.3 \\ -70.6 & -45.3 \\ -41 * * & -38 * *\end{array}$

$\begin{array}{lcrcrc}\theta_{+}(\mathrm{mV}) & A_{\mathrm{LTD}}\left(\mathrm{mV}^{-1}\right) & A_{\mathrm{LTD}}\left(\mathrm{mV}^{-2}\right) & \tau_{x}(\mathrm{~ms}) & \tau_{-}(\mathrm{ms}) & \tau_{+}(\mathrm{ms}) \\ -45.3 & 14 \times 10^{-5 * *} & 8 \times 10^{-5 * *} & 15^{* *} & 10^{* *} & 7^{* *} \\ -45.3 & 21 \times 10^{-5 * *} & 30 \times 10^{-5 * *} & 30^{* *} & 6 * * & 5^{* *} \\ -38 * * & 38 \times 10^{-5 * *} & 2 \times 10^{-5 * *} & 16 & & \end{array}$

(a) Parameters for the neuron model. (b) Plasticity rule parameters for the various experiments. * indicates the standard set of parameters. ${ }^{* *}$ indicates the free parameters fitted to experimental data. Other parameters were set in advance on the basis of previous studies. 
groups of neurons had formed, which were characterized by strong bidirectional connectivity in the group, and different receptive fields and no lateral connectivity between groups (Fig. 5c). If the overall amplitude of plastic changes was small (compared with that found in the experiments), the pattern of lateral connectivity was stable and had only a few strong bidirectional connections amidst a great deal of weak lateral connectivity. The reason for this is that two neurons with similar receptive fields are both active at high rate whenever the stimulus is in the center region of their receptive field, which gives rise to strong bidirectional lateral connections (Fig. 4). Unidirectional strong connections were nearly absent (Fig. 5). If the amplitude and rate of plasticity is more realistic and consistent with our data (Fig. 2), then the pattern of lateral connectivity changed between one snapshot in time and another one $5 \mathrm{~s}$ later, but the overall pattern was stable when averaged over $100 \mathrm{~s}$ (Fig. 5f-h). In each snapshot, about half of the strong connections were bidirectional (Fig. $5 \mathbf{h}, \mathbf{i}$ ).

This connectivity pattern contrasts with that shown under a temporal coding procedure (Fig. 6). Neurons developed receptive fields similar to those seen with the rate-coding procedure, but, as expected for temporal Hebbian learning ${ }^{14}$, the receptive field shifted over time (Fig. 6a). With a small learning rate, this shift was slow, as in previous models ${ }^{14}$, but with realistic learning parameters extracted from our experiments (Fig. 2), the shift of the receptive field was rapid (Fig. 6a). Notably, among the lateral connections, strong reciprocal links were nearly absent, whereas strong unidirectional connections from neuron $n$ to neurons $n+1, n+2$ and $n+3$ dominated (Fig. 6b-e). As the pattern of feedforward connections forming the receptive fields changed, the structure of lateral connections changed as well on the time scale of $10 \mathrm{~min}$. Nevertheless, at each moment in time, the pattern of lateral connections was highly asymmetric, favoring connections from neuron $n$ to $n+k$ (with $k=1,2$ and 3 ) over those from $n$ to $n-k$, where $n$ is the neuronal index after relabeling according to the receptive field position (Fig. 6a). This suggests that temporal coding procedures in which stimuli are nonstationary and exhibit systematic spatio-temporal correlations are reflected in the functional connectivity pattern by strong unidirectional connections, whereas rate coding (characterized by stationary input with spatial correlations only) leads to strong bidirectional connections. We confirmed this for a broad range of stimuli and in the presence of noise (Supplementary Figs. 1-3).

\section{Development of localized receptive fields}

The results for the feedforward connectivity in the previous subsection lead to the question of the behavior of our plasticity model under stimulation procedures previously used for rate models ${ }^{24,34,35}$. Both our spiking rule and the rate-based $\mathrm{BCM}$ model ${ }^{24}$ require presynaptic activity to induce a change. Furthermore, for our rule, as well as for the simplest BCM rule (see ref. 24), the depression terms are linear and the potentiation terms are quadratic in the postsynaptic variables (that is, the postsynaptic potential or the postsynaptic firing rate). More quantitatively, for Poisson input, the total weight change $\Delta w$ in our model is proportional to $v^{\text {pre }} v^{\text {post }}\left(v^{\text {post }}-\vartheta\right)$, where $v^{\text {pre }}$ and $v^{\text {post }}$ denote the firing rates of pre- and postsynaptic neurons, respectively, and $\vartheta$ is a sliding threshold related to the ratio between the LTP- and LTD-inducing processes (equation (8)). The sliding threshold arises in our plasticity model because the amount of LTD $A_{\text {LTD }}$ depends on the long-term average of the voltage on the slow time scale of homeostatic processes. Because of its similarities to BCM, we were not surprised that our spike-based learning rule with sliding threshold was able to support the development of localized receptive fields, a feature related to independent component analysis (ICA) and sparse coding ${ }^{24,34}$.
In our experiments, the input consists of small patches of natural images using standard preprocessing ${ }^{36}$. After learning with our plasticity rule, the weights exhibit a stable spatial structure that can be interpreted as a receptive field (Fig. 7). In contrast with a principal component analysis of image patches (as, for example, implemented by Hebbian learning in linear neurons ${ }^{37}$ ), the receptive fields were localized (that is, the region with strong weights did not stretch across the whole image patch). Nine runs of the learning experiments gave receptive fields with different locations and orientations (Fig. 7d). Because of the homeostatic control of LTD in our plasticity model, the neuron compensated in experiments with increased input firing rates by developing smaller receptive fields that were even more localized (Fig. 7e). Development of localized receptive fields has been interpreted as a signature of ICA or sparse coding ${ }^{35}$. In contrast with most other ICA algorithms ${ }^{36}$, our rule is biologically more plausible, as it is consistent with data from a large body of plasticity experiments.

\section{DISCUSSION}

Because traditional plasticity rules are rate models, the relation between coding and connectivity cannot be studied. Our plasticity rule is formulated on the level of postsynaptic voltage. Because action potentials are sharp voltage peaks, they act as singular events in the voltage so that, in the presence of a spike, our rule turns automatically into a spike timing-dependent rule. Indeed, for spike coding (and without subthreshold voltage manipulations), our plasticity rule behaves similar to a STDP rule in which triplets of spikes with pre-post-post or postpre-post timing evoke $\mathrm{LTP}^{26,27}$, whereas pairs with post-pre timing evoke LTD. In contrast with standard STDP rules (reviewed in ref. 14), pairing-frequency dependence ${ }^{16}$ and burst-timing dependence ${ }^{30}$ are qualitatively described. In addition, the rule is expected to reproduce the triplet and quadruplet experiments in hippocampal slices ${ }^{38}$ (data not shown), as for all STDP protocols the plasticity rule that we used is similar to an earlier nonlinear STDP rule ${ }^{27}$. Deriving STDP rules from voltage dependence has been attempted before ${ }^{16,39,40}$. However, because these earlier models use the momentary voltage $\mathrm{e}^{40}$ or its derivative ${ }^{39}$, rather than the combination of momentary and averaged voltage that we used in our model, these earlier models cannot account for the broad range of nonlinear effects in STDP experiments or interaction of voltage and spike timing. The voltage-based model ${ }^{16}$ uses separate empirical functions for timing dependence, voltage dependence, frequency dependence and multiple spike summation with preference for LTP to capture the nonlinear effects of LTP. Our model is similar in that it also uses momentary voltage before the spike as one of the variables, but it requires neither an explicit frequencydependent term nor an explicit timing-dependent term. Instead, frequency and timing dependence follow from the model dynamics. Our model has similarities with LTP induction in the TagTriC model $^{41}$, but the TagTriC model focuses on the long-term stability of synapses, rather than spike-timing dependence of the induction mechanism.

Even though our model does not require a biophysical interpretation of the variables, it is tempting to speculate about potential mechanisms. For the depression term in our model, a trace $\bar{u}_{-}$left by previous activity of the postsynaptic neuron is combined with spike arrival $x$ at the presynaptic terminal (Fig. 1a). In light of the results on LTD in layer V neocortical neurons ${ }^{42}$, this trace could be related to endocannabinoids released from the postsynaptic site. Coincidence of this slow trace with the activation of presynaptic NMDA receptors (which rapidly respond to the glutamate released by presynaptic activity $x(t))$ could be the trigger signal for $\mathrm{LTD}^{42}$. Indeed, the duration of the LTD component in the STDP function increases if the endocannabinoid trace is artificially prolonged (see Fig. 9 of 
ref. 42). In other neuron types and brain areas, the same mathematical model (but with different parameters) could correspond to different biophysical mechanisms of LTD. For example, in hippocampal CA1 neurons, the trace $\bar{u}_{-}$could reflect calcium entry through voltagegated ion channels during depolarization, which, when combined with synaptic signals (caused by the presynaptic spike arrival $x$ ), would give rise to the calcium signals that are necessary to trigger LTD (reviewed in refs. 17,18,42). Potentiation is induced in our model by the combination of three factors: a momentary depolarization above spike threshold, a depolarization just before the spike, $\bar{u}_{+}$, above rest, and the presence of a trace $\bar{x}$ left by presynaptic spike arrival (Fig. 1a). The trace $\bar{x}$ could correspond to the amount of glutamate bound to the postsynaptic NMDA receptor, but this is controversial ${ }^{42}$. A high momentary voltage $u$ can be induced by a backpropagating action potential; notably, backpropagation of action potentials is more likely to occur and will more reliably occur in the background of a weak depolarization of the dendrite ${ }^{42}$, and such a weak depolarization potentially corresponds to the term $\bar{u}_{+}$in our model. Because we have a depolarizing afterpotential after each spike in our model (Fig. 1c,d), the value of $\bar{u}$ just before the next spike increases with the repetition frequency of the STDP protocol, consistent with previous experiments (Fig. 5d in ref. 42). Our model is therefore consistent with previous results showing that LTP can be induced in distal synapses only if additional cooperative input or dendritic depolarization prevents failure of backpropagating action potentials ${ }^{43}$. In the context of the classical view of the NMDA receptor as a coincidence detector $^{42}$, it is quite natural to see why a sequence post-pre-post of two postsynaptic action potentials and one presynaptic spike are ideal for LTP. The spike afterpotential of the first postsynaptic action potential removes the calcium block and prepares the dendrite for successful backpropagation of a later action potential. If the backpropagating action potential caused by the second postsynaptic spike occurs just slightly after presynaptic spike arrival, this causes a sharply peaked and large calcium transient that would be sufficient to trigger the LTP induction chain.

Even though our model is formulated on the level of voltage, we do not imply that voltage itself is the essential biophysical mechanism. Rather, under physiological conditions, the voltage transient (or current or conductance transient) caused by synaptic input or action potential firing is the starting point of long biochemical signaling chains that lead to induction of plasticity. In our phenomenological model, the signature of the inputs (here, voltage transients) are directly linked (via mathematical variables or traces) to the induction of plasticity, jumping over the biophysical mechanisms of the signal transduction chain.

Our plasticity rule allows us to explain experiments from two different studies with a single principle. Both the 'potentiation is rescued by depolarization'16 scenario (Fig. 2f) and that of burst-timing dependent $\operatorname{LTP}^{30}$ (Fig. 3 ) indicate that LTP is induced at low frequency when the membrane is depolarized before the pre-post pairing. This depolarization can be the result of a previous spike during a postsynaptic burst ${ }^{30}$ or to a depolarization current. A further unexpected result is that, with the set of parameters derived from visual cortex slice experiments, synapses fluctuated rapidly between strong and weak weights. This aspect is interesting in light of the synapse mobility that has been reported in imaging experiments ${ }^{5}$.

Possible extensions of the model include a weight dependence of synaptic plasticity. We assumed that weights can grow to a hard upper bound, but the rule can easily be changed to soft bounds ${ }^{14}$ by changing the prefactors $A_{\text {LTP }}$ and $A_{\text {LTD }}$ accordingly ${ }^{41}$. Second, short-term plasticity ${ }^{44}$ could be added for a better description of the plasticity phenomena that occurs during high-frequency protocols. Third, additional mechanisms need to be implemented to describe the transition from early to late LTP/LTD ${ }^{41,45}$. Finally, we can generalize from point neurons to spatially extended neurons using a multicompartment neuron model (for example, distinct compartments for the soma and dendrites). We did not do this here because detailed spatial models introduce a considerable number of new parameters, making overfitting more likely to occur. Notably, our voltage-based formulation of plasticity, if applied locally in a compartmental model, would allow potentiation to occur in a dendritic branch whenever the three conditions-presynaptic activity, recent postsynaptic depolarization and momentary large depolarization-occur together, independent of the source of depolarization. Thus, dendritic spikes could lead to potentiation in the absence of somatic action potentials, consistent with data from experiments in hippocampal ${ }^{46-48}$ and cortical slices ${ }^{31}$.

Our plasticity model leads to several predictions that could be tested in slice experiments. First, the model predicts that in voltage-clamp experiments the weight change is dependent on the voltage and the number of presynaptic spikes but not on their exact timing (for example, low frequency, tetanus or burst). Second, in the scenario in which potentiation is rescued by depolarization, the amount of weight change should be the same whether a depolarizing current of amplitude B stops precisely when the postsynaptic spike is triggered or whether a current of slightly bigger amplitude $\mathrm{B}^{\prime}$ stops a few milliseconds earlier.

The influence of STDP on temporal coding has been previously studied with respect to changes in the feedforward connections (reviewed in ref. 14). The effect of STDP on lateral connectivity has been much less studied ${ }^{20-23}$. We found that, because of STDP, coding influences the network topology; that is, different stimulation procedures generate different patterns of lateral connectivity. Our results contrast with those of standard STDP rules, which always suppress short loops and, in particular, bidirectional connections ${ }^{19,32}$. Our more realistic plasticity model shows that under a rate-coding procedure (where the neuron is stimulated by different stationary patterns), bidirectional connectivity and highly connected clusters with multiple loops are not only possible but even dominant. It is only for temporal coding (characterized by stimulation with substantial spatiotemporal correlations) that our biologically plausible rule leads to dominant unilateral directions. We speculate that the differences in coding between different brain areas could lead, even if the learning rule were exactly the same, to different network topologies. Our model predicts that experiments in which cells in a recurrent network are repeatedly stimulated in a fixed order would decrease the fraction of strong bidirectional connections, whereas a stimulation pattern in which clusters of neurons fire at a high rate during episodes of a few hundred milliseconds would increase this fraction. In this view, it is tempting to connect the low degree of bidirectional connectivity in barrel cortex ${ }^{4}$ to the bigger importance of temporal structure in whisker input ${ }^{33}$, compared with visual input ${ }^{3}$.

\section{METHODS}

Methods and any associated references are available in the online version of the paper at http://www.nature.com/natureneuroscience/.

Note: Supplementary information is available on the Nature Neuroscience website.

\section{ACKNOWLEDGMENTS}

This work was supported by the European project FACETS and the Swiss National Science Foundation.

\section{AUTHOR CONTRIBUTIONS}

C.C. developed the model and carried out the experiments. L.B. and E.V. participated in discussions. W.G. supervised the project and wrote most of the manuscript. 


\section{COMPETING INTERESTS STATEMENT}

The authors declare no competing financial interests.

Published online at http://www.nature.com/natureneuroscience/.

Reprints and permissions information is available online at http://www.nature.com/ reprintsandpermissions/.

1. Buonomano, D.V. \& Merzenich, M.M. Cortical plasticity: from synapses to maps. Annu. Rev. Neurosci. 21, 149-186 (1998).

2. Dan, Y. \& Poo, M. Spike timing-dependent plasticity of neural circuits. Neuron 44, 23-30 (2004)

3. Song, S., Sjöström, P.J., Reigl, M., Nelson, S. \& Chklovskii, D.B. Highly nonrandom features of synaptic connectivity in local cortical circuits. PLOS Biol. 3, e350 (2005).

4. Lefort, S., Tomm, C., Sarria, J.C.F. \& Petersen, C.C.H. The excitatory neuronal network of the $\mathrm{C} 2$ barrel column in mouse primary somatosensory cortex. Neuron 61, 301-316 (2009)

5. Yuste, R. \& Bonhoeffer, T. Genesis of dendritic spines: insights from ultrastructural and imaging studies. Nat. Rev. Neurosci. 5, 24-34 (2004).

6. Hebb, D.O. The Organization of Behavior (Wiley, New York, 1949).

7. Malenka, R.C. \& Bear, M.F. LTP and LTD: an embarassment of riches. Neuron 44, 5-21 (2004).

8. Markram, H., Lübke, J., Frotscher, M. \& Sakmann, B. Regulation of synaptic efficacy by coincidence of postsynaptic APs and EPSPs. Science 275, 213-215 (1997).

9. Artola, A., Bröcher, S. \& Singer, W. Different voltage-dependent thresholds for inducing long-term depression and long-term potentiation in slices of rat visual cortex. Nature 347, 69-72 (1990).

10. Ngezahayo, A., Schachner, M. \& Artola, A. Synaptic activity modulates the induction of bidirectional synaptic changes in adult mouse hippocampus. J. Neurosci. 20, 2451-2458 (2000).

11. Dudek, S.M. \& Bear, M.F. Bidirectional long-term modification of synaptic effectiveness in the adult and immature hippocampus. J. Neurosci. 13, 2910-2918 (1993).

12. Gerstner, W., Kempter, R., Van Hemmen, L. \& Wagner, H. A neuronal learning rule for sub-millisecond temporal coding. Nature 383, 76-81 (1996).

13. Legenstein, R., Naeger, C. \& Maass, W. What can a neuron learn with spike timingdependent plasticity? Neural Comput. 17, 2337-2382 (2005).

14. Gerstner, W. \& Kistler, W.M. Spiking Neuron Models (Cambridge University Press, New York, 2002)

15. Lisman, J. \& Spruston, N. Postsynaptic depolarization requirements for LTP and LTD: a critique of spike timing-dependent plasticity. Nat. Neurosci. 8, 839-841 (2005).

16. Sjöström, P.J., Turrigiano, G.G. \& Nelson, S.B. Rate, timing and cooperativity jointly determine cortical synaptic plasticity. Neuron 32, 1149-1164 (2001).

17. Shouval, H.Z., Bear, M.F. \& Cooper, L.N. A unified model of NMDA receptor dependent bidirectional synaptic plasticity. Proc. Natl. Acad. Sci. USA 99, 10831-10836 (2002)

18. Lisman, J.E. \& Zhabotinsky, A.M. A model of synaptic memory: a CaMKII/PP1 switch that potentiates transmission by organizing an AMPA receptor anchoring assembly. Neuron 31, 191-201 (2001).

19. Song, S. \& Abbott, L.F. Cortical development and remapping through spike timingdependent plasticity. Neuron 32, 339-350 (2001).

20. Lubenov, E.V. \& Siapas, A.G. Decoupling through synchrony in neuronal circuits with propagation delays. Neuron 58, 118-131 (2008).

21. Levy, N., Horn, D., Meilijson, I. \& Ruppin, E. Distributed synchrony in a cell assembly of spiking neurons. Neural Netw. 14, 815-824 (2001).

22. Morrison, A., Aertsen, A. \& Diesmann, M. Spike timing-dependent plasticity in balanced random networks. Neural Comput. 19, 1437-1467 (2007).

23. Izhikevich, E.M. \& Edelman, G.M. Large-scale model of mammalian thalamocortical systems. Proc. Natl. Acad. Sci. USA 105, 3593-3598 (2008).

24. Cooper, L.N., Intrator, N., Blais, B.S. \& Shouval, H.Z. Theory of Cortical Plasticity (World Scientific, Singapore, 2004).
25. Miller, K.D. A model for the development of simple cell receptive fields and the ordered arrangement of orientation columns through activity dependent competition between ON- and OFF-center inputs. J. Neurosci. 14, 409-441 (1994).

26. Senn, W., Tsodyks, M. \& Markram, H. An algorithm for modifying neurotransmitter release probability based on pre- and postsynaptic spike timing. Neural Comput. 13, 35-67 (2001).

27. Pfister, J.-P. \& Gerstner, W. Triplets of spikes in a model of spike timing-dependent plasticity. J. Neurosci. 26, 9673-9682 (2006)

28. O'Connor, D.H., Wittenberg, G.M. \& Wang, S.S.H. Dissection of bidirectional synaptic plasticity into saturable unidirectional processes. J. Neurophysiol. 94 1565-1573 (2005).

29. Turrigiano, G.G. \& Nelson, S.B. Homeostatic plasticity in the developing nervous system. Nat. Rev. Neurosci. 5, 97-107 (2004).

30. Nevian, T. \& Sakmann, B. Spine $\mathrm{Ca}^{2+}$ signaling in spike timing-dependent plasticity. J. Neurosci. 26, 11001-11013 (2006).

31. Kampa, B.M., Letzkus, J.J. \& Stuart, G.J. Requirement of dendritic calcium spikes for induction of spike timing-dependent synaptic plasticity. J. Physiol. (Lond.) 574 283-290 (2006).

32. Kozloski, J. \& Cecchi, G.A. Topological effects of synaptic spike timing-dependent plasticity. Preprint at <http://arxiv.org/abs/0810.0029> (2008).

33. Jadhav, S.P., Wolfe, J. \& Feldman, D.E. Sparse temporal coding of elementary tactile features during active whisker sensation. Nat. Neurosci. (2009).

34. Blais, B.S., Intrator, N., Shouval, H. \& Cooper, L. Receptive field formation in natural scene environments. Comparison of single-cell learning rules. Neural Comput. 10, 1797-1813 (1998).

35. Olshausen, B.A. \& Field, D.J. Emergence of simple-cell receptive field properties by learning a sparse code for natural images. Nature 381, 607-609 (1996).

36. Hyvärinen, A., Karhunen, J. \& Oja, E. Independent Component Analysis (Wiley, New York, 2001).

37. Oja, E. A simplified neuron as a principal component analyzer. J. Math. Biol. 15, 267-273 (1982)

38. Wang, H.X., Gerkin, R.C., Nauen, D.W. \& Bi, G.-Q. Coactivation and timing dependent integration of synaptic potentiation and depression. Nat. Neurosci. 8 187-193 (2005).

39. Saudargiene, A., Porr, B. \& Wörgötter, F. How the shape of pre- and postsynaptic signals can influence STDP: a biophysical model. Neural Comput. 16, 595-626 (2004).

40. Brader, J.M., Senn, W. \& Fusi, S. Learning real-world stimuli in a neural network with spike-driven synaptic dynamics. Neural Comput. 19, 2881-2912 (2007).

41. Clopath, C., Ziegler, L., Vasilaki, E., Büsing, L. \& Gerstner, W. Tag-trigger consolidation: a model of early and late long-term potentiation and depression. PLOS Comput. Biol. 4, e1000248 (2008).

42. Sjöström, P.J., Turrigiano, G.G. \& Nelson, S.B. Neocortical LTD via coincident activation of presynaptic NMDA and cannabinoid receptors. Neuron 39, 641-654 (2003).

43. Sjöström, P.J. \& Häusser, M. A cooperative switch determines the sign of synaptic plasticity in distal dendrites of neocortical pyramidal neurons. Neuron $51,227-238$ (2006).

44. Tsodyks, M.V. \& Markram, H. The neural code between neocortical pyramidal neurons depends on neurotransmitter release probability. Proc. Natl. Acad. Sci. USA 94, 719-723 (1997).

45. Frey, U. \& Morris, R.G.M. Synaptic tagging and long-term potentiation. Nature $\mathbf{3 8 5}$ 533-536 (1997)

46. Remy, S. \& Spruston, N. Dendritic spikes induce single-burst long-term potentiation PNAS 104, 17192-17197 (2007).

47. Hardie, J. \& Spruston, N. Synaptic depolarization is more effective than back propagating action potentials during induction of associative long-term potentiation in hippocampal pyramidal neurons. J. Neurosci. 29, 3233-3241 (2009).

48. Golding, N.L., Staff, N.P. \& Spruston, N. Dendritic spikes as a mechanism for cooperative long-term potentiation. Nature 418, 326-331 (2002). 


\section{ONLINE METHODS}

Neuron model. In contrast with standard models of STDP, our plasticity model uses the postsynaptic membrane potential $u(t)$. As a model for neuronal voltage, we chose the adaptive exponential integrate-and-fire (AdEx) mode ${ }^{49}$ with an additional current describing the depolarizing spike after potential ${ }^{50}$. The voltage evolution is

$$
C \frac{d}{d t} u=-g_{L}\left(u-E_{L}\right)+g_{L} \Delta_{T} e^{\frac{u-V_{T}}{\Delta_{T}}}-w_{a d}+z+I
$$

where $C$ is the membrane capacitance, $g_{L}$ is the leak conductance, $E_{L}$ is the resting potential and $I$ is the stimulating current. The exponential term describes the activation of sodium current. The parameter $\Delta_{T}$ is the slope factor and $V_{T}$ is the threshold potential. A hyperpolarizing adaptation current is described by the variable $w_{a d}$ with dynamics

$$
\tau_{w_{a d}} \frac{d}{d t} w_{a d}=a\left(u-E_{L}\right)-w_{a d}
$$

where $\tau_{w_{a d}}$ is the time constant of the adaption of the neuron and $a$ is a parameter. On firing, the variable $u$ is reset to the fixed value $V_{\text {reset }}$, whereas $w_{a d}$ is increased by the amount $b$. The main difference between this and a previously described model $^{23}$ is that the voltage is exponential rather than quadratic, allowing for a better fit to data ${ }^{50}$. The spike afterpotential of the cells used in typical STDP experiments ${ }^{16}$ have a long depolarizing spike afterpotential. We therefore added an additional current $z$, which is set to a value $I_{\text {sp }}$ immediately after a spike occurs and decays otherwise with a time constant $\tau_{z}$

$$
\tau_{z} \frac{d}{d t} z=-z
$$

Finally, refractoriness was modeled with the adaptive threshold $V_{T}$, which starts at $V_{T_{\max }}$ after a spike and decays to $V_{T_{\text {rest }}}$ with a time constant $\tau_{V_{T}}{ }^{50}$, that is,

$$
\tau_{V_{T}} \frac{d}{d t} V_{T}=-\left(V_{T}-V_{T_{\text {rest }}}\right)
$$

Parameters for the neuron model are taken from ref. 49 for the AdEx model, $\tau_{z}$ was set to $40 \mathrm{~ms}$, consistent with ref. 16 (see also ref. 50), and kept fixed throughout all simulations (see Table 1a).

Plasticity model. Our model exhibits separate additive contributions to the plasticity rule, one for LTD and another one for LTP ${ }^{28}$. For the LTD part, we assumed that presynaptic spike arrival at synapse $i$ induces depression of the synaptic weight $w_{i}$ by $-A_{\mathrm{LTD}}\left(\bar{u}_{-}(t)-\theta_{-}\right)_{+} \cdot()_{+}$indicate rectification, that is, any value $\bar{u}_{-}<\theta_{-}$does not lead to a change ${ }^{9}$ (see Fig. $\mathbf{1 h}$ ). The quantity $\bar{u}_{-}(t)$ is an exponential low-pass-filtered version of the postsynaptic membrane potential $u(t)$ with time constant $\tau$

$$
\tau_{-} \frac{d}{d t} \bar{u}_{-}(t)=-\bar{u}_{-}(t)+u(t)
$$

The variable $\bar{u}_{-}$is an abstract variable that could, for example, reflect the level of calcium concentration ${ }^{17}$ or the release of endocannabinoids ${ }^{42}$, although such an interpretation is not necessary for our rule. Because the presynaptic spike train is described as a series of short pulses at time $t_{i}^{n}$, where $i$ is the index of the synapse and $n$ an index that counts the spike $X_{i}(t)=\sum_{n} \delta\left(t-t_{i}^{n}\right)$, depression is represented by

$$
\frac{d}{d t} w_{i}^{-}=-A_{\mathrm{LTD}}(\overline{\bar{u}}) X_{i}(t)\left(\bar{u}_{-}-\theta_{-}\right)_{+} \text {if } w_{i}>w_{\min }
$$

where $A_{\mathrm{LTD}}(\overline{\bar{u}})$ is an amplitude parameter that is under the control of homeostatic process ${ }^{29}$. For slice experiments, the parameter has a fixed value that was determined experimentally. For the network simulations shown in Figures 5-7, the parameter depends on the mean depolarization $\bar{u}_{-}$of the postsynaptic neuron, averaged over a time scale of $1 \mathrm{~s}$. Equation (1) is a simple method for implementing homeostasis; other methods, such as weight rescaling, would also be possible ${ }^{29}$. The time scale of $1 \mathrm{~s}$ is not critical ( $100 \mathrm{~s}$ or more would be more realistic for homeostasis) but is convenient for the numerical implementation.
For the LTP component, we assumed that each presynaptic spike at the synapse $w_{i}$ increases the trace $\bar{x}_{i}(t)$ of some biophysical quantity, which decays exponentially with a time constant $\tau_{x}^{12,27}$

$$
\tau_{x} \frac{d}{d t} \bar{x}_{i}(t)=-\bar{x}_{i}(t)+X_{i}(t)
$$

where $X_{i}(t)$ is the spike train defined above. The quantity $\bar{x}_{i}(t)$ could, for example, represent the amount of glutamate bound to postsynaptic receptors ${ }^{27}$ or the number of NMDA receptors in an activated state ${ }^{26}$. Potentiation is given by

$$
\frac{d}{d t} w_{i}^{+}=A_{\mathrm{LTP}} \bar{x}_{i}\left(u-\theta_{+}\right)_{+}\left(\bar{u}_{+}-\theta_{-}\right)_{+} \quad \text { if } w_{i}<w_{\max }
$$

Here, $A_{\mathrm{LTP}}$ is a free amplitude parameter fitted to the data and $\bar{u}_{+}(t)$ is another low-pass-filtered version of $u(t)$ that is similar to $\bar{u}_{-}(t)$ but has a shorter time constant $\tau_{+}$of around $10 \mathrm{~ms}$. Thus, positive weight changes can occur if the momentary voltage $u(t)$ surpasses a threshold $\theta_{+}$and, at the same time, the average value $\bar{u}_{+}(t)$ is above $\theta_{-}$.

The final rule used in the simulation was

$$
\frac{d}{d t} w_{i}=-A_{\mathrm{LTD}}(\overline{\bar{u}}) X_{i}\left(\bar{u}_{-}-\theta_{-}\right)_{+}+A_{\mathrm{LTP}} \bar{x}_{i}\left(u-\theta_{+}\right)_{+}\left(\bar{u}_{+}-\theta_{-}\right)_{+}
$$

combined with the hard bounds $w_{\min } \leq w_{i} \leq w_{\max }$. For network simulation, we used $A_{\mathrm{LTD}}(\overline{\bar{u}})=A_{\mathrm{LTD}} \frac{\overline{\bar{u}}^{2}}{u_{\text {ref }}^{2}}$, where $u_{\text {ref }}^{2}$ is a reference value.

It is unlikely that the model can be simplified further. First, voltage is necessary as a variable whenever voltage is manipulated in experiments. Second, dependence on voltage must be nonlinear ${ }^{9-11}$. Phenomenological models have some freedom in the choice of the mathematical form of the nonlinearities (for example, exponential, polynomial Hill functions or piecewise linear) and we chose a suitable combination of piecewise linear functions with thresholds $\theta_{+}$and $\theta_{-}$. Third, STDP experiments indicate that the temporal relation between stimulation events is important. All timing relations have been implemented as (first order) linear filtering. For the case of classical STDP experiments, where all spikes are triggered by the experimenter, our phenomenological model can be simplified and becomes identical or closely related to existing nonlinear STDP model ${ }^{26,27}$, but regarding the interaction between voltage and spike timing, such a further simplification is not possible. Finally, the fact that the curve of burst timing-dependent plasticity (Fig. 3c) is not perfectly reproduced indicates that our plasticity model does not have an unnecessarily large number of free parameters.

Analysis of plasticity model. We established a quantitative link between our plasticity model (equation (3)) and BCM theory ${ }^{24}$ under the assumption of a linear Poisson neuron model with spikes. In a linear Poisson neuron, input spike trains $X_{j}(t)=\sum_{i} \delta\left(t-t_{i}^{f}\right)$ are low-pass filtered and weighted to give a subthreshold potential $u^{s}(t)=\sum_{j} \int_{0}^{\infty} \varepsilon(s) X_{j}(t-s) d s$, where $\varepsilon(s)$ is the time course of an excitatory postsynaptic potential and $u^{s}$ is measured with respect to the resting potential $\theta_{-}$. The linear Poisson neuron generates spikes stochastically with stochastic firing intensity $v^{\text {post }}$ proportional (with parameter $1 / \alpha$ ) to $u^{s}$, hence the probability of firing in a short time between $t$ and $t+\Delta$ is $P_{F}(t ; t+\Delta)=v^{\text {post }}(t) \Delta=u^{s}(t) \frac{\Delta}{\alpha}$. If the linear Poisson neuron spikes at time $t_{f}^{\text {post }}$, we add a short voltage pulse $\beta \delta\left(t-t_{f}^{\text {post }}\right)$. The total membrane potential is therefore

$$
u(t)=u^{s}(t)+\beta Y(t)+\theta_{-}
$$

where $Y(t)=\sum_{f} \delta\left(t-t_{f}^{\text {post }}\right)$ is the spike train of the postsynaptic neuron and

$\beta$ is the integral weight of spikes. To illustrate the importance of $\beta$, suppose that in a hypothetical experiment of $100-\mathrm{ms}$ duration we found a single triangular action potential with amplitude $120 \mathrm{mV}$ and a 1-ms duration at halfmaximum, and that the voltage was otherwise constant at a value of $2 \mathrm{mV}$ above rest. The mean voltage averaged over this $100-\mathrm{ms}$ period would therefore be 
$\int_{0}^{100} \frac{u(t) d t}{100}=2 \mathrm{mV}+1.2 \mathrm{mV}+\theta_{-}$so that the weight parameter $\beta$ in equation (4) should have a value of $1.2 \mathrm{mV}$.

By construction, the expected number of spikes of the linear Poisson neuron is equal to its instantaneous rate $\left\langle Y>(t)=v^{\text {post }}(t)=\frac{u^{s}(t)}{\alpha}\right.$. In the following derivation, the time dependence of the variables is not explicitly denoted for the sake of simplicity (except for a few special cases); for example, $u(t)$ is abbreviated as $u$.

We assumed that the neuron has $N$ excitatory synapses stimulated by $N$ presynaptic Poisson spike trains of rates $v^{\text {pre }}=\left(v^{\text {pre }}, \ldots, v_{N 1}^{\text {pre }}\right)$. Furthermore, we assumed that the presynaptic rates $v^{\text {pre }}$ are slowly varying quantities compared with the intrinsic time scales $\tau_{+}$and $\tau_{-}$of our plasticity model or those of our neuron model (for example, excitatory postsynaptic potential duration), which were all below $50 \mathrm{~ms}$. This assumption explicitly resulted in the following simplifications: $\bar{v}^{\text {pre }} \approx v^{\text {pre }}, \bar{v}_{-}^{\text {post }} \approx v^{\text {post }}$ and $\bar{v}_{+}^{\text {post }} \approx v^{\text {post }}$. For a variable $q, \bar{q}$ denotes low-pass filtering with the time constant $\tau_{q}$, and $\bar{q}_{+}$and $\bar{q}_{-}$correspond to the time constants $\tau_{+}$and $\tau_{-}$, respectively.

Using the linear Poisson model defined above in the plasticity rule (equation (3)) yields (if we suppress for the moment the dependence on the homeostatic variable $\overline{\bar{u}}$ )

$$
\frac{d}{d t} w_{i}=-A_{\mathrm{LTD}} X_{i}(t)\left(\bar{u}_{-}^{s}+\beta \bar{Y}_{-}\right)+A_{\mathrm{LTP}} \bar{x}_{i}(t) \beta Y\left(\bar{u}_{+}^{s}+\beta \bar{Y}_{+}\right)
$$

with $\theta$ being equal to the resting potential, all voltages being above resting potential, as only excitatory inputs are considered, and only $Y$ being above the firing threshold $\theta_{+}$, as $u^{s}$ was the subthreshold voltage. Taking the average $<$. $>_{\text {post }}$ over the postsynaptic spikes given the postsynaptic rate $v^{\text {post }}$ yields

$<\frac{d}{d t} w_{i}>_{\text {post }}=-(\alpha+\beta) A_{\mathrm{LTD}} X_{i}(t) \bar{v}_{-}^{\text {post }}+(\alpha+\beta) A_{\mathrm{LTP}} \bar{x}_{i}(t) \beta v^{\text {post }} \bar{v}_{+}^{\text {post }}$

Here, we used $\left\langle Y(t) \bar{Y}_{+}(t)\right\rangle_{\text {post }}=v^{\text {post }}(t) \bar{v}_{+}^{\text {post }}(t)$, which holds because $\bar{Y}_{+}(t)$ is not influenced by a possible spike at time $t$ (just by spikes at times $s$ with $s<t$ ), and it is therefore uncorrelated with $Y(t)$ given $v^{\text {post }}$. For slowly varying input rates

$<\frac{d}{d t} w_{i}>_{\text {post }}=-(\alpha+\beta) A_{\mathrm{LTD}} X_{i}(t) v^{\text {post }}+(\alpha+\beta) A_{\mathrm{LTP}} \bar{x}_{i}(t) \beta v^{\text {post }} v^{\text {post }}$

Taking the average $\langle\text {. }\rangle_{\text {post }}$ over the presynaptic spikes given the presynaptic firing rates $v^{\text {pre }}$ and neglecting spike-spike correlations (that is, correlations between $X_{i}$ and $v^{\text {post }}$ beyond rate correlations between $v^{\text {pre }}$ and $v^{\text {post }}$ ) gives

$$
\begin{aligned}
<\frac{d}{d t} w_{i}>_{\text {post }} & =-(\alpha+\beta) A_{\mathrm{LTD}} v_{\mathrm{i}}^{\text {pre }} v^{\text {post }}+(\alpha+\beta) A_{\mathrm{LTP}} v_{\mathrm{i}}^{\text {pre }} \beta v^{\text {post }} v^{\text {post }} \\
& =(\alpha+\beta) \beta A_{\mathrm{LTP}} v_{\mathrm{i}}^{\text {pre }} v^{\text {post }}\left(v^{\text {post }}-\frac{A_{\mathrm{LTD}}}{\beta A_{\mathrm{LTP}}}\right)
\end{aligned}
$$

Here, $<<.{ }_{\text {post }}>_{\text {pre }}$ was abbreviated as $<.>$. The factor $(\alpha+\beta) \beta$ can be interpreted as the learning rate in a rate-based plasticity model and $\frac{A_{\mathrm{LTD}}}{\beta A_{\mathrm{LTP}}}=\vartheta$ as the threshold for the transition of LTD to LTP in the quadratic BCM model ${ }^{24}$. Because $A_{\text {LTD }}$ depends on the slow time scale of homeostatic processes on the long-term averaged potential $\overline{\bar{u}}$, the threshold $\vartheta$ is a sliding one. Just as in the BCM model ${ }^{24}$, our plasticity model responds to persistent periods of high activity with an increase in the threshold $\vartheta$.

Parameters and data fitting. For the plasticity slice experiments, we took $\overline{\bar{u}}=u_{\text {ref }}$ as fixed and fit the parameter $A_{\mathrm{LTD}}$. The total number of parameters of the plasticity model is therefore seven. For all data sets, except the one taken from ref. 10, the threshold $\theta_{-}$was set to the resting potential and $\theta_{+}$to the firing threshold of the AdEx model, that is, $\theta_{-}=-70.6 \mathrm{mV}$ and $\theta_{+}=-45.3 \mathrm{mV}$. The remaining five parameters, $\tau_{x}, \tau_{-}, \tau_{+}, A_{\text {LTD }}$ and $A_{\text {LTP }}$ were fitted to each data set individually by the following procedure. We calculated the theoretically predicted weight change $\Delta w_{i}^{\text {th, } j}$ by integrating (analytically or numerically) equation (3), for a given experimental protocol $j$, as a function of the free parameters. We then estimated the free parameters by minimizing the mean-square error $E$ between the theoretical calculations and the experimental data $\Delta w_{i}^{\text {exp }, j}$

$$
E=\sum_{j}\left(\Delta w_{i}^{\mathrm{th}, j}-\Delta w_{i}^{\mathrm{exp}, j}\right)^{2}
$$

For the data set in hippocampus ${ }^{10}$, we also fit the two parameters $\theta_{-}$and $\theta_{+}$, as completely different preparations and cell type were used. Moreover, for this data set, the time constant $\tau_{x}$ was taken from physiological measurements given in ref. 2 and fixed to the value of $16 \mathrm{~ms}$. The parameters for the various experiments are summarized in Table $\mathbf{1 b}$.

Voltage-clamp experiment. The postsynaptic membrane potential was switched in the simulations to a constant value, $u_{\text {clamp }}$, chosen from -80 to $0 \mathrm{mV}$ while synapses were stimulated with either 25 (blue line) or 100 pulses (red line) at $50 \mathrm{~Hz}$. As a result of voltage clamping, the actual value of the voltage $u$ itself and the low-pass-filtered versions $\bar{u}$ are constant and equal to $u_{\text {clamp }}$. Thus, the synaptic plasticity rule becomes

$$
\frac{d}{d t} w_{i}=-A_{\mathrm{LTD}} X_{i}(t)\left(u_{\text {clamp }}-\theta_{-}\right)_{+}+A_{\mathrm{LTP}} \bar{x}_{i}(t)\left(u_{\text {clamp }}-\theta_{+}\right)_{+}\left(u_{\text {clamp }}-\theta_{-}\right)_{+} .
$$

STDP experiment and frequency dependence. Presynaptic spikes in the simulation were paired with postsynaptic spikes that were either advanced by $+10 \mathrm{~ms}$ or delayed by $-10 \mathrm{~ms}$ with respect to the presynaptic spike. Postsynaptic spikes were triggered by brief, strong current pulses into the postsynaptic neuron. The pairing was repeated five times with different frequencies ranging from 0.1 to $50 \mathrm{~Hz}$. These five pairings were repeated 15 times at $0.1 \mathrm{~Hz}$. However, the five pairings at $0.1 \mathrm{~Hz}$ were repeated only ten times to mimic the experimental protocol ${ }^{16}$.

Burst timing-dependent plasticity For Figure 3a, the presynaptic spike was paired $\Delta t=+10 \mathrm{~ms}$ before (or $\Delta t=-10 \mathrm{~ms}$ after) 1,2 or 3 postsynaptic spikes. The frequency of the burst was $50 \mathrm{~Hz}$. The neuron received 60 pairings at a frequency of $0.1 \mathrm{~Hz}$. For Figure $3 \mathbf{b}$, the presynaptic spike was paired with a burst of three action potentials $(\Delta t=+10 \mathrm{~ms}$ and $-10 \mathrm{~ms}$ ), whereas the burst frequency varies from 20 to $100 \mathrm{~Hz}$. For Figure 3c, a presynaptic spike is paired with a burst of three postsynaptic action potentials with burst frequency of $50 \mathrm{~Hz}$. The time $\Delta t$ between the presynaptic spike and the first postsynaptic action potential varies from -80 to $40 \mathrm{~ms}$. For a detailed description of the experiments, see ref. 30 .

Poisson input for functional scenarios. Poisson inputs were used in all of the following experiments. They were generated by a stochastic process where the spike was elicited with a stochastic intensity $v$.

Relation between connectivity and coding: toy model. Weights of ten all-to-all connected neurons were initialized at 1 , bounded between 0 and 3 . Weights evolved with the voltage-based rule (equation (3)) for $100 \mathrm{~s}$. The model was compared with a canonical pair-based STDP model written as $\frac{d}{d t} w_{i}=-A_{\mathrm{LTD}}^{\mathrm{pair}} X_{i} \bar{y}+A_{\mathrm{LTP}}^{\text {pair }} \bar{x}_{i} Y$, where $Y$ is the postsynaptic spike train defined in the same manner as the presynaptic spike train $X_{i}$ with a filter of the postsynaptic spikes $\bar{y}$ similar to $\bar{x}_{i}$.

We chose the parameters $A_{\mathrm{LTD}}^{\text {pair }}=A_{\mathrm{LTP}}^{\text {pair }}=1 \times 10^{-5}$ for the amplitudes and $\tau_{x}$ for the time constant of $\bar{x}_{i}$, as well as for the time constant of the postsynaptic low-pass filter $\bar{y}$. Neuron 1 fired at $2 \mathrm{~Hz}$, neuron 2 at $4 \mathrm{~Hz}$. neuron 10 at $20 \mathrm{~Hz}$ following Poisson statistics; that is, short current pulses were injected to make the neuron fire with Poisson statistics at this frequency. Neurons fired successively every $20 \mathrm{~ms}$, with neuron 1 firing, followed $20 \mathrm{~ms}$ later by neuron $2, \ldots$ followed by neuron 10 and then back to neuron 1 , etc. in a loop.

Rate coding in network simulation. 500 presynaptic Poisson neurons with firing rates $v_{i}^{\text {pre }}(1 \leq i \leq 500)$ are connected to 10 postsynaptic excitatory neurons. The input rates $v_{i}^{\text {pre }}$ follow a Gaussian profile, that is, $v_{i}^{\text {pre }}=A e^{\frac{-(i-\mu)^{2}}{2 \sigma^{2}}}$, with variance $\sigma=10$ and amplitude $A=30 \mathrm{~Hz}$. The center $\mu$ of the Gaussian shifts randomly every $100 \mathrm{~ms}$ between ten different, equally distributed positions. 
Circular boundary conditions are assumed, that is, neuron $i=500$ is considered to be a neighbor of $i=1$. Synaptic weights of the feedforward connections to the excitatory neurons are initialized randomly (uniformly in $[0.5,2]$ ) and hard bound are set to 0 and 3 . The ten excitatory neurons are all-to-all recurrently connected with a starting synaptic weight of 0.25 (hard bounds set to 0 and 0.75 ). In addition, three inhibitory neurons are driven by eight excitatory neurons and the feedforward inputs; they project onto six excitatory neurons, connectivity chosen randomly. Those random recurrent connections are fixed and have a weight equal to 1 . The feedforward connections onto the inhibitory neurons are also fixed and chosen randomly between 0 and 0.5 . The reference value is set to $u_{\text {ref }}^{2}=60 \mathrm{mV}^{2}$ and the simulation time to $1,000 \mathrm{~s}$. Parameters were normally chosen as in Table $\mathbf{1 b}$ (visual cortex data), except for Fig. 5b-e, where $A_{\text {LTP }}$ and $A_{\text {LTD }}$ were reduced by a factor 100 .

Temporal coding in network simulation. Settings were determined as described above, but patterns were presented for $20 \mathrm{~ms}$ successively (from center position 50 to 100 to 150 , etc. in a circular manner). The reference value was set to $u_{\text {ref }}^{2}=80 \mathrm{mV}^{2}$. We used an asymmetry index calculated by relabeling the neurons according to the current position of their receptive field so that with the cyclic stimulation they get activated one after the other: $n \rightarrow n+1 \ldots \rightarrow n+k \rightarrow n-1$ $\rightarrow n$. We then compared the connection from $n$ to $n+k$ with that from $n$ to $n$

$-k$ and computed $A S=\sum_{k} w_{n, n+k}-w_{n, n-k}, k=1-3$. Connectivity patterns were also analyzed in model networks where neurons received (in addition to feedforward and lateral input) unspecific stochastic background activity that made them fire spontaneously (Supplementary Fig. 1).

ICA-like computation: orientation selectivity with natural images. Ten natural images have been taken from a previously determined benchmark ${ }^{35}$. A small patch of $16 \times 16$ pixels from any of the images is randomly chosen every $200 \mathrm{~ms}$, which is on the order of the fixation time between saccades. Half of the time the image matrix is transposed, flipped around the vertical axis or the horizontal axis to remove any statistical orientation bias. After prewhitening, the inputs for the ON (OFF) image are Poisson spike trains generated by the positive (negative) part of the patch (with respect to a reference gray value reflecting the ensemble mean) with maximum frequency of $50 \mathrm{~Hz}$. The $2 \times 16 \times 16$ inputs are connected to one postsynaptic neuron. The initial weights are set randomly between 0 and 2 and hard bounds are set between 0 and 3 . The connections follow the synaptic rule (equation (3)), where the reference value is set to $u_{\mathrm{ref}}^{2}=50 \mathrm{mV}^{2}$. Parameters were chosen as in Table $\mathbf{1 b}$ (visual cortex data), but $A_{\mathrm{LTP}}$ and $A_{\mathrm{LTD}}$ were reduced by a factor 10 . Every $20 \mathrm{~s}$, an extra normalization was applied to equalize the norm of the ON weights to one of the OFF weights ${ }^{25}$.

49. Brette, R. \& Gerstner, W. Adaptive exponential integrate-and-fire model as an effective description of neuronal activity. J. Neurophysiol. 94, 3637-3642 (2005).

50. Badel, L. et al. Dynamic I-V curves are reliable predictors of naturalistic pyramidalneuron voltage traces. J. Neurophysiol. 99, 656-666 (2008). 